\title{
A National Survey of Medical Oncologist's Opinions and Perceptions for Managing Rash Among mCRC Patients Treated with Panitumumab
}

Kimberly A. Lowe $\cdot$ Laura Sangaré $\cdot$ Rachel Bergstresser •

Michelle McNamara · George Kafatos · Tamer Garawin

Received: February 27, 2019 / Published online: May 3, 2019

(C) The Author(s) 2019

\section{ABSTRACT}

Introduction: This study aimed to describe medical oncologist's opinions and perceptions regarding the management of dermatologic toxicities among metastatic colorectal cancer (mCRC) patients who were treated with panitumumab in the USA and assess if there were differences across demographic and clinical characteristics.

Methods: We developed a survey based on the current literature and expert opinions regarding the management of dermatologic toxicities. The survey was implemented online in September 2016. Eligible oncologists were board certified and had treated at least five new or continuing patients with mCRC in the last 3 months, among whom at least three patients had

Enhanced Digital Features To view enhanced digital features for this article go to https://doi.org/10.6084/ m9.figshare.7931510.

K. A. Lowe $(\bowtie) \cdot$ T. Garawin

Amgen, Inc, Thousand Oaks, CA, USA

e-mail: lowek@amgen.com

L. Sangaré

SimulStat, Portland, OR, USA

R. Bergstresser · M. McNamara

Adelphi Research, Doylestown, PA, USA

G. Kafatos

Amgen, Ltd, Cambridge, UK received or were currently receiving panitumumab.

Results: A total of 250 oncologists completed the survey. The data suggest that approximately $82 \%$ of patients received recommendations for moisturizer, $88 \%$ for sunscreen and $67 \%$ for ultraviolet (UV)-protective garments prior to or at the time of initiation of panitumumab therapy. There were minor differences in how dermatologic toxicities were managed across specific demographic or clinical groups. The data also suggest that the management associated with panitumumab use among mCRC patients can be greatly improved.

Conclusions: Our results highlight the urgent need for heightened education regarding dermatologic toxicity management among oncologists who treated mCRC patients with panitumumab. Easily implemented strategies, such as moisturizer, sunscreen, and UV-protective garments should be recommended to all patients.

Funding: Amgen, Inc.

Plain Language Summary: Plain language summary available for this article.

Keywords: Acneiform rash; Anti-EGFR; Dermatologic toxicity; Metastatic colorectal cancer; Panitumumab 


\section{PLAIN LANGUAGE SUMMARY}

Panitumumab is a medication for the treatment of metatstatic colorectal cancer. This medication frequently causes a painful rash. However, management strategies exist that are more likely to keep the rash in a mild form. If not managed properly, there is a risk that the rash can progress to a more moderate or severe form which can affect a patient's quality of life.

\section{What do Oncologists Currently Know About Strategies to Best Manage Rash Associated with Panitumumab?}

To find out, we asked medical oncologist's their opinions and perceptions regarding the management of skin toxicities caused from treatment with panitumumab.

We identified three common problems in how oncologists currently manage rash:

1. Managing rash should start at the time of the first dose of panitumumab, but oncologists typically wait until the rash emerges before providing treatment with prescription or other-the-counter medications.

2. Not all patients receive the recommendations for the most basic and effective management strategies, including use of moisturizer, sunscreen, and UV-protective garments, such as hats.

3. Oncologists do not typically involve a dermatologist to help manage patient's rash.

Oncologists need more education on how rash associated with panitumumab can be most effectively managed. A well-informed oncologist can better inform their patients on how to prepare for treatment to minimize the risk of a more burdensome rash. Easily implemented strategies, such as moisturizer, sunscreen, and UV-protective garments, should be recommended to all patients.

\section{INTRODUCTION}

In 2017, an estimated 135,000 Americans were newly diagnosed with colorectal cancer (CRC), adding to the approximately 1.5 million
Americans who are alive with a history of CRC [1]. Approximately 50\% of CRC patients will eventually develop metastatic colorectal cancer (mCRC), and an estimated $20-25 \%$ of new cases have mCRC at diagnosis [2-4]. Among cancers, CRC has the fourth highest mortality rate in the USA, with an annual mortality rate that is currently estimated at 14.1 deaths per 100,000, representing approximately 50,000 deaths annually $[1,5]$. The mortality rate due to CRC has dropped $51 \%$ since its peak of 28.6 per 100,000 in 1976 [1]. Increased survival rates have been attributed to both earlier detection and treatment improvements [1]. mCRC treatments targeting the epidermal growth factor receptor (EGFR), namely panitumumab (Vectibix $^{\circledR}$ ) and cetuximab (Erbitux $\left.{ }^{\circledR}\right)$, account for some of the improvements in survival and are the focus of this paper [6].

Acute and chronic skin toxicities are commonly experienced among mCRC patients who are treated with anti-EGFR therapies. The prototypical cutaneous adverse reaction associated with all EGFR inhibitors is acneiform eruption, which represents the most common form of acute dermatologic toxicity. The acneiform rash occurs in approximately $80 \%$ of patients who are treated with an anti-EGFR; its severity is usually grade $1-2$, although $15-20 \%$ of patients experience acute toxicity (grade 3 or higher) [7-9]. The rash typically occurs early in the course of therapy and is associated with pruritus and pain; presentation of the rash leads to dose reduction or treatment cessation as well as impaired quality of life in approximately $30 \%$ of patients who are treated with an anti-EGFR $[7,9,10]$.

Despite the high incidence of dermatologic toxicities in this patient population, there are currently no clinical standards for how these patients are managed even though clinical standards do exist. There is clear evidence from the Skin Toxicity Evaluation Protocol with Panitumumab (STEPP) [11] and the Japan Skin Toxicity Evaluation Protocol with Panitumumab (J-STEPP) [12] studies that the severity of the rash can be significantly reduced if it is managed pre-emptively. In these studies, the pre-emptive skin treatment regimen began 1 day before the first panitumumab dose and 
continued through weeks 1 to 6 . The regimen consisted of skin moisturizer, sunscreen, 1\% hydrocortisone cream, and doxycycline $100 \mathrm{mg}$ twice per day in the STEPP study, and skin moisturizer, sunscreen, $0.5 \%$ hydrocortisone cream, and minocycline $100 \mathrm{mg}$ once per day in the J-STEPP study. Both studies demonstrated reduced severity in panitumumab-associated dermatologic toxicities through the implementation of pre-emptive versus reactive skin management.

Improving the manner in which rash is managed among mCRC patients who are treated with anti-EGFR therapies first requires an understanding of how it is currently managed using real-world data. This information is currently lacking. Therefore, the aim of our study was to describe medical oncologist's opinions and perceptions regarding the management of dermatologic toxicities among mCRC patients who were treated with panitumumab in the USA and to assess if there were differences across demographic and clinical characteristics. These data may provide insights into interventions that can increase the utilization of pre-emptive management strategies, thereby reducing the incidence and severity of skin toxicity associated with antiEGFR therapies among mCRC patients.

\section{METHODS}

\section{Online Survey}

This was a cross-sectional study that utilized an online survey that was distributed to oncologists in the USA in September 2016. The 30-min survey included questions on demographic characteristics of the physicians, as well as opinions on how dermatologic toxicities are typically managed among mCRC patients, and specifically what agents they recommend (i.e., moisturizers, sunscreen, ultraviolet [UV]-protective garments, over-the-counter topical steroids, prescription steroids, topical antibiotics, and oral antibiotics) and when they recommend them relative to the onset of rash. There were specific questions related to treatment background (five questions), general skin toxicity management (nine questions), specifics of skin toxicity management (nineteen questions), and therapy adjustment (eleven questions). The survey was developed using expert opinions and current literature and underwent two rounds of pilot testing to ensure readability, sensibility and content validity.

\section{Participants}

To be eligible to participate in the study, physicians needed to be board certified in oncology. They also needed to have treated at least five new or continuing mCRC patients in the previous 3 months, of whom at least three of these patients must have received, or were still be receiving, therapy with an EGFR inhibitor. Exclusion criteria included (1) failing to provide informed consent; and (2) not allowed to be compensated for participation in survey research (i.e., those who are licensed in Vermont and those who treat patients in Government or Department of Veterans Affairs settings).

\section{Stratification by Participant Demographics}

Given the objectives of the study, all analyses were stratified based on the following demographic and clinical characteristics of the participating oncologist: region within the USA (west, midwest, south, or northeast), practice type (academic or community-based practice), years of practice ( $\leq 10$ years or $>10$ years), and practice size ( $\leq 5$ or $>5$ doctors).

\section{Data Analysis}

Data analysis was performed using STATA version 10.0 (StataCorp 2007; StataCorp LP, College Station, TX, USA). This was a descriptive study. Responses were cross-tabulated and compared based on region in the USA, practice type, years in practice, and practice size.

\section{Compliance with Ethics Guidelines}

All procedures performed in studies involving human participants were in accordance with 
the Quorum Review institutional review board in the US and with the 1964 Helsinki declaration and its later amendments or comparable ethical standards. Informed consent was obtained from all individual participants included in the study.

\section{RESULTS}

The demographic and clinical expertise of the 250 medical oncologists who completed the survey is summarized in Table 1 . The practice locations of the participants were evenly distributed across the USA. The majority of the participants reported practicing at community cancer centers $(n=160,64 \%)$ and were practicing for $>10$ years $(n=151,60 \%)$.

\section{Skin Toxicity Management Strategies and Timing}

Based on analysis of the returned survey forms, approximately $82 \%$ of mCRC patients were receiving recommendations for moisturizer, $88 \%$ for sunscreen, and $67 \%$ for UV-protective garments prior to or at the time of panitumumab initiation. The proportion of participants who recommended each of the skin toxicity management strategies, stratified by the participant's demographics, is depicted in Fig. 1. There were minor differences in how participants reported managing rash across the demographic groups of interest. The small descriptive trends that emerged from the data included: (1) a slightly higher percentage of participants in the northeast reporting using skin moisturizer and sunscreen than participants in other regions (Fig. 1a); (2) a slightly higher percentage of participants who practiced at community cancer centers reported using skin moisturizer and sunscreen than did participants who practiced at academic centers (Fig. 1b); (3) a slightly higher proportion of participants who had been practicing for $\leq$ 10 years reported recommending each treatment management strategy (with the exception of over-the-counter [OTC]-strength topical steroids) than did participants who had been practicing $>10$ years (Fig. 1c); (4) and a slightly higher proportion of participants who practiced with $\leq 5$ doctors reported recommending each treatment management strategy (with the exception of OTC-strength topical steroids) than did participants who had been practicing for $>5$ years (Fig. $1 \mathrm{~d}$ ).

The timing of skin management treatment initiation as it related to treatment with panitumumab is described in Table 2 . The timing of skin toxicity treatments were similar across the demographic groups of interest. In general, a higher proportion of participants across all of the demographic groups reported initiating the use of skin moisturizer, sunscreen, and UVprotective garments prior to or at the same time as initiation of treatment with panitumumab than with other treatment regimens, compared to other treatment options. OTC-strength topical steroids, prescription-strength steroids, topical antibiotics, and oral antibiotics were most commonly recommended at the first sign of any rash or later across all demographic groups.

\section{Utilization of Nursing Support and Dermatology}

Utilization of nursing support to assist in the management of skin toxicity was common in this cohort of oncologists (Table 3). Participants in the western USA consistently reported utilizing nursing care less than did participants in other regions of the USA. When asked about the frequency with which they consulted a dermatologist, the most common response (approximately 40\%) was "occasionally," regardless of their demographics (Table 3).

\section{DISCUSSION}

Acneiform rash has a significant negative impact on a patient's quality of life both emotionally and physically, and effective management of skin toxicity is known to improve the quality of life of patients $[7,13,14]$. In addition, dermatologic toxicities are known to result in cessation of anti-EGFR therapies or dose 


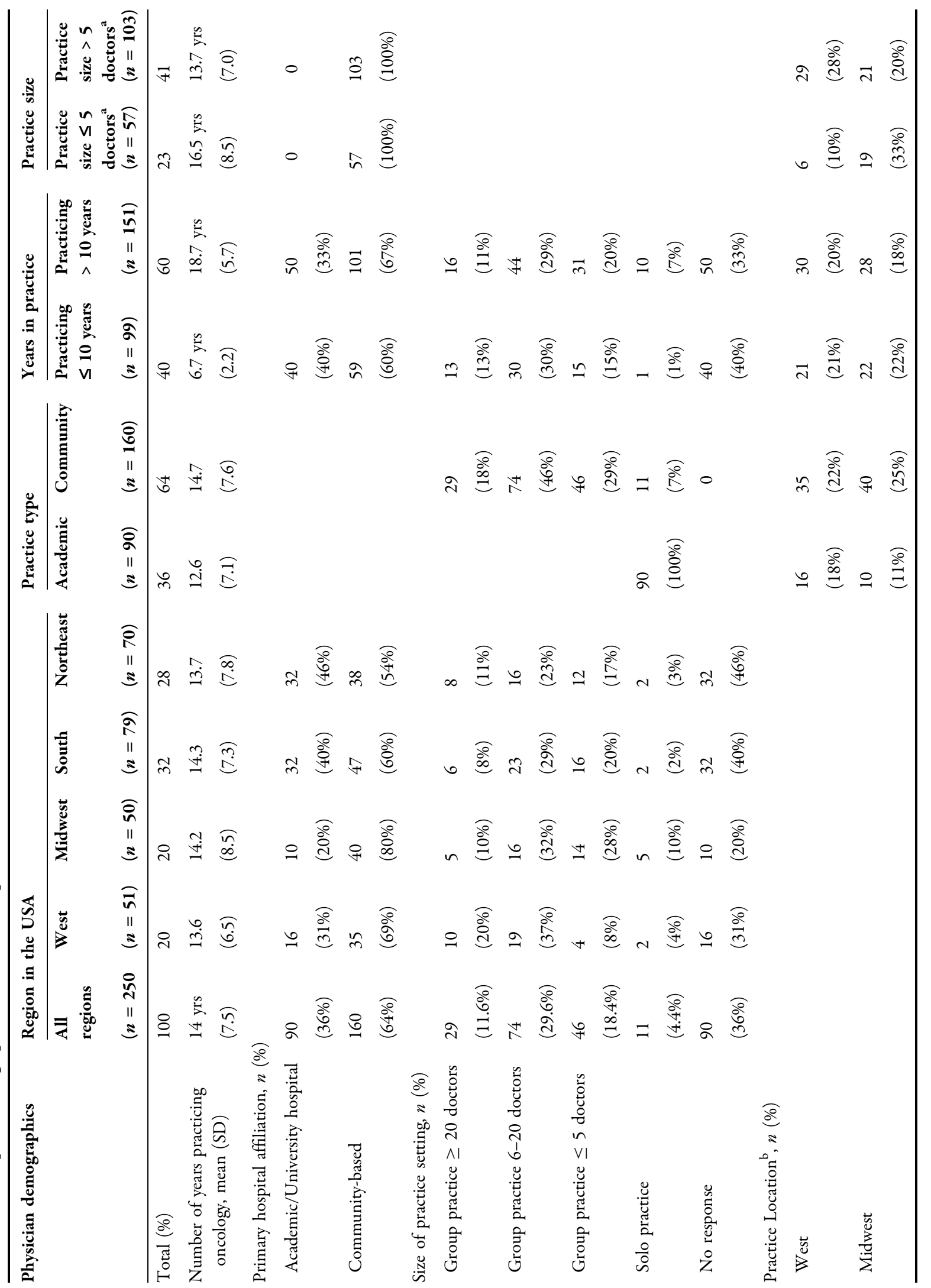




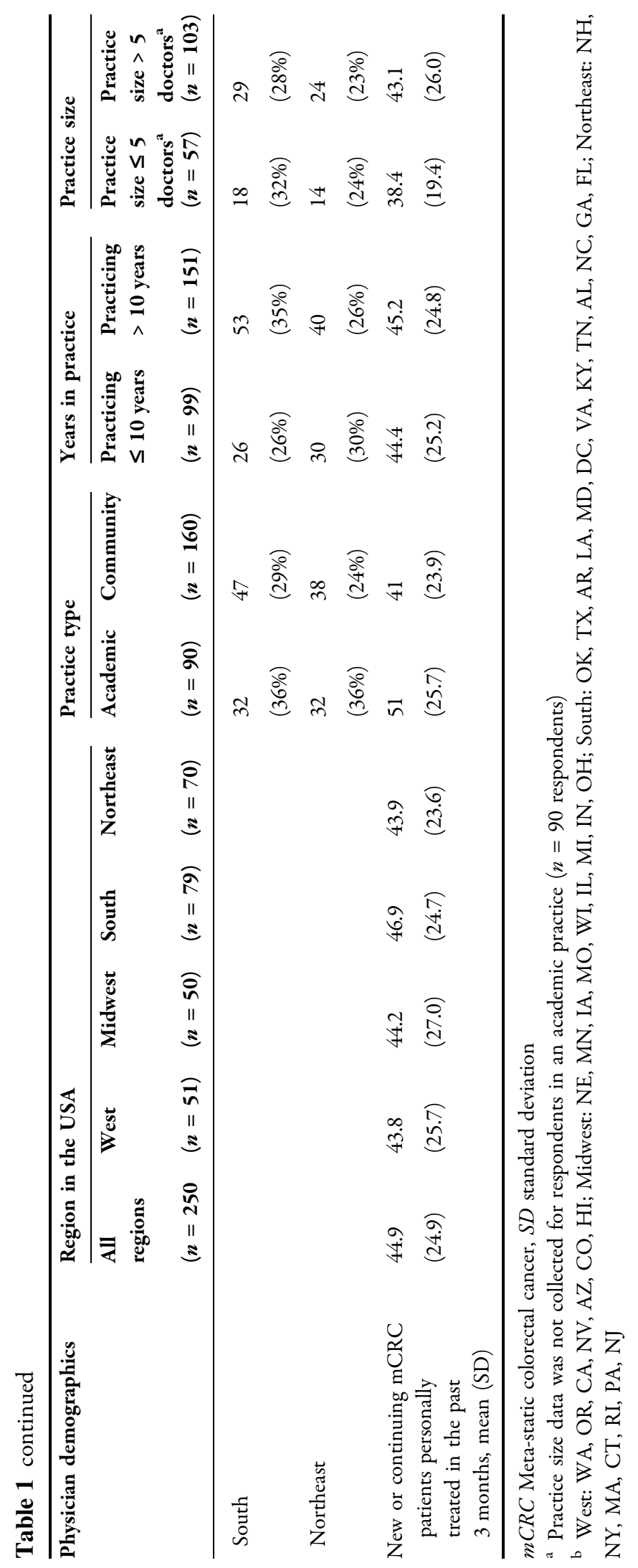



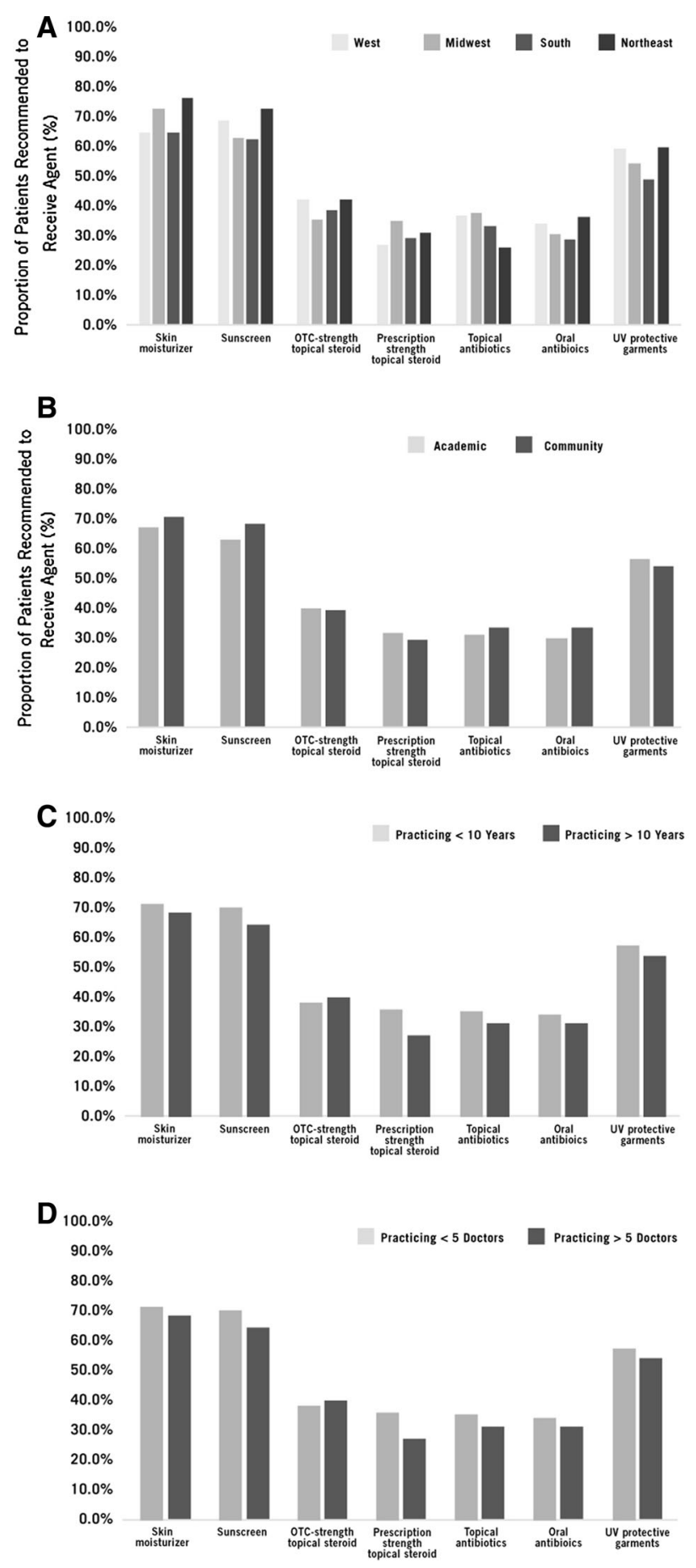

Fig. 1 Proportion of patients who were recommended each of the skin toxicity management strategies, stratified by the participant's demographics. OTC over-the-counter, $U V$ ultraviolet

reductions, both of which can negatively impact efficacy of the therapy and survival of the patient $[7,14]$.
The results from this study suggest management practices for the treatment of skin toxicity associated with panitumumab use among 


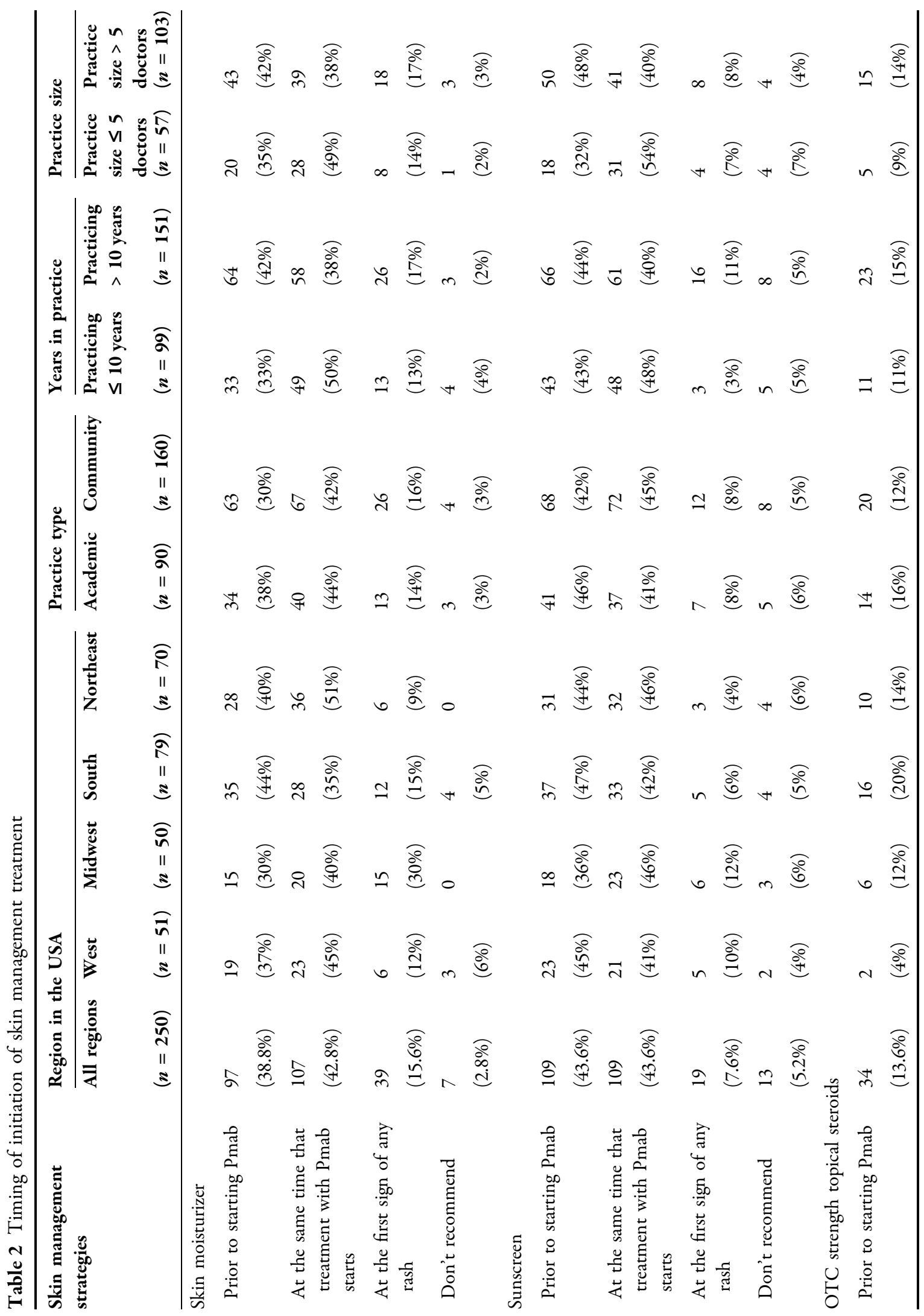




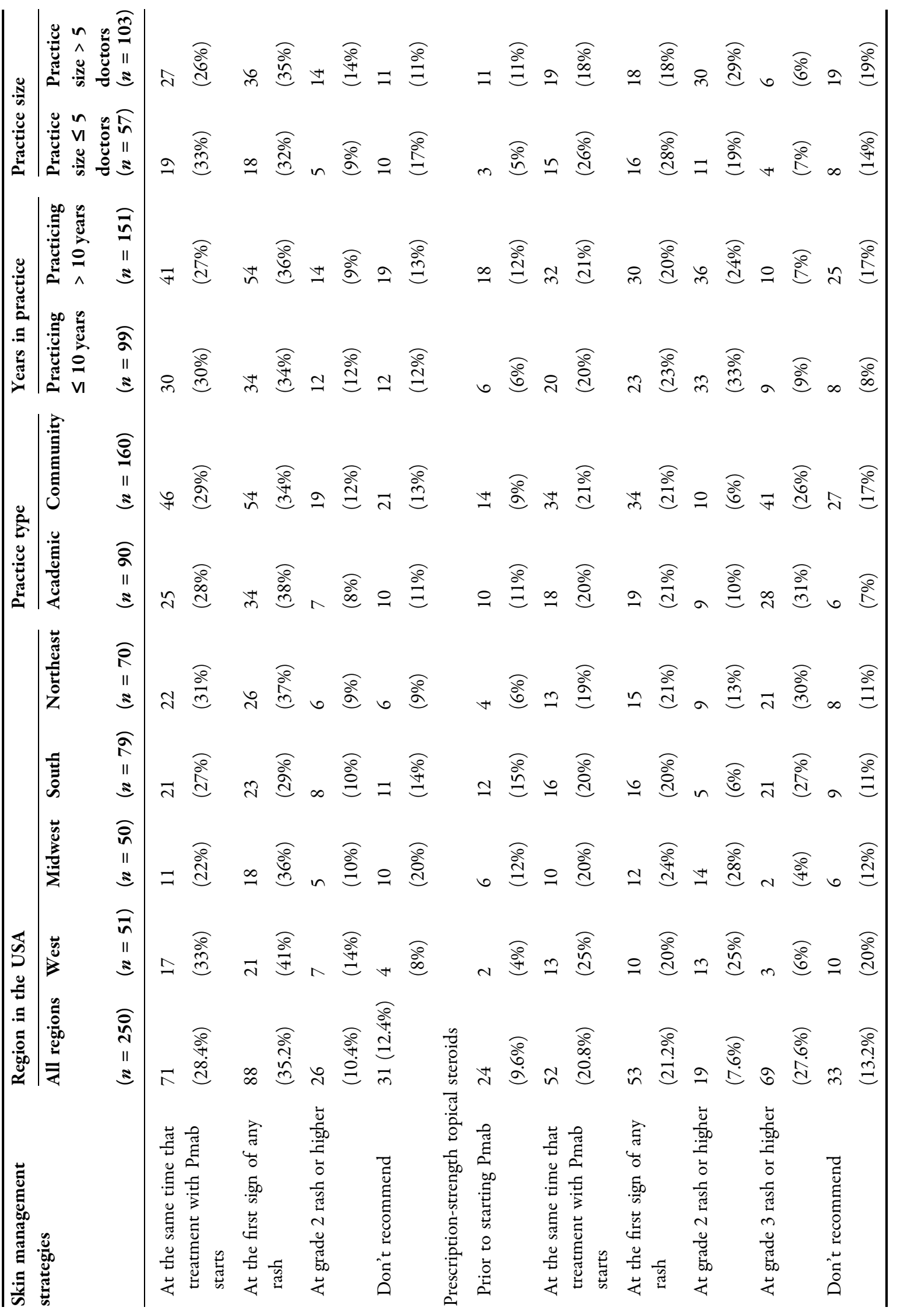




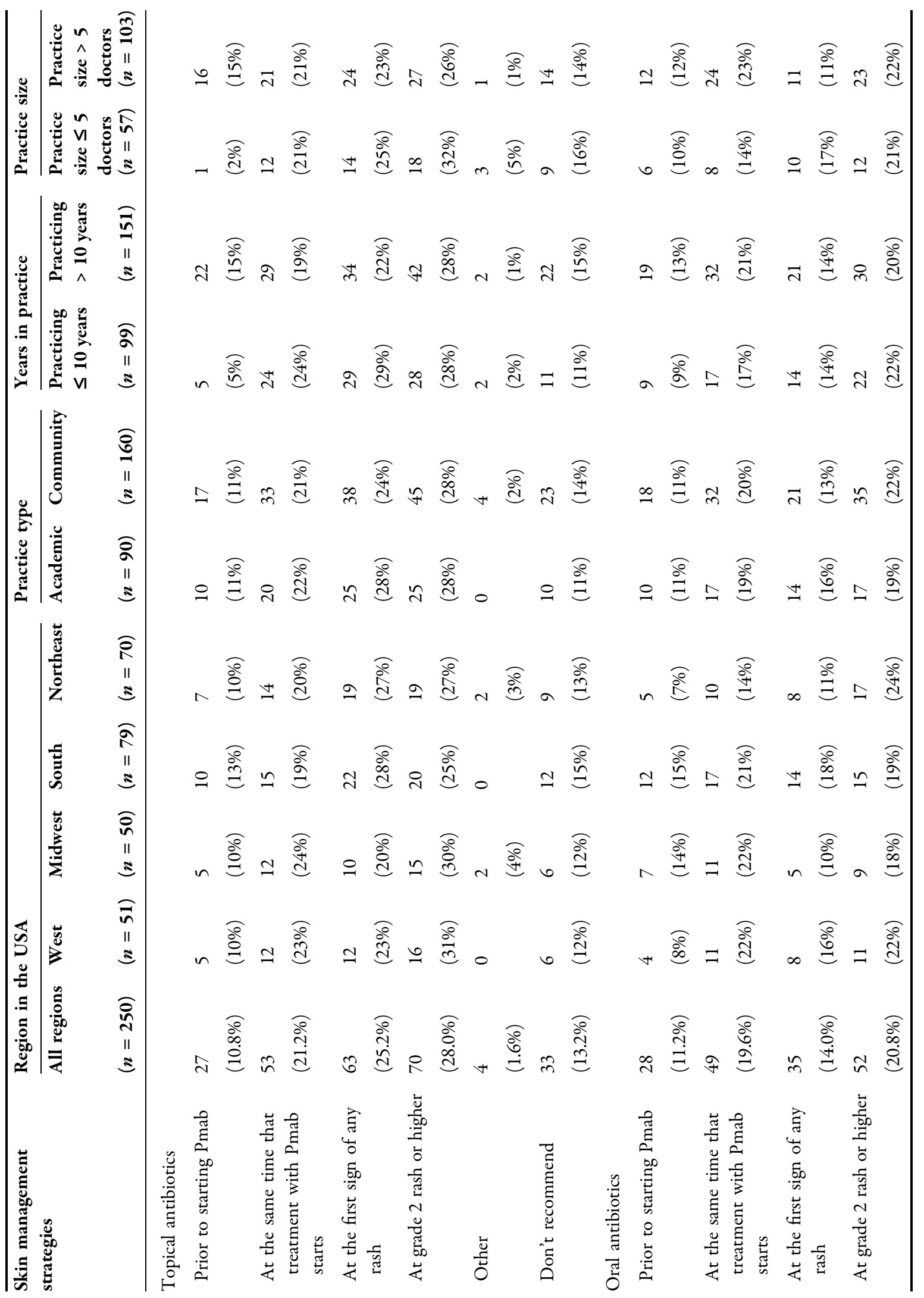




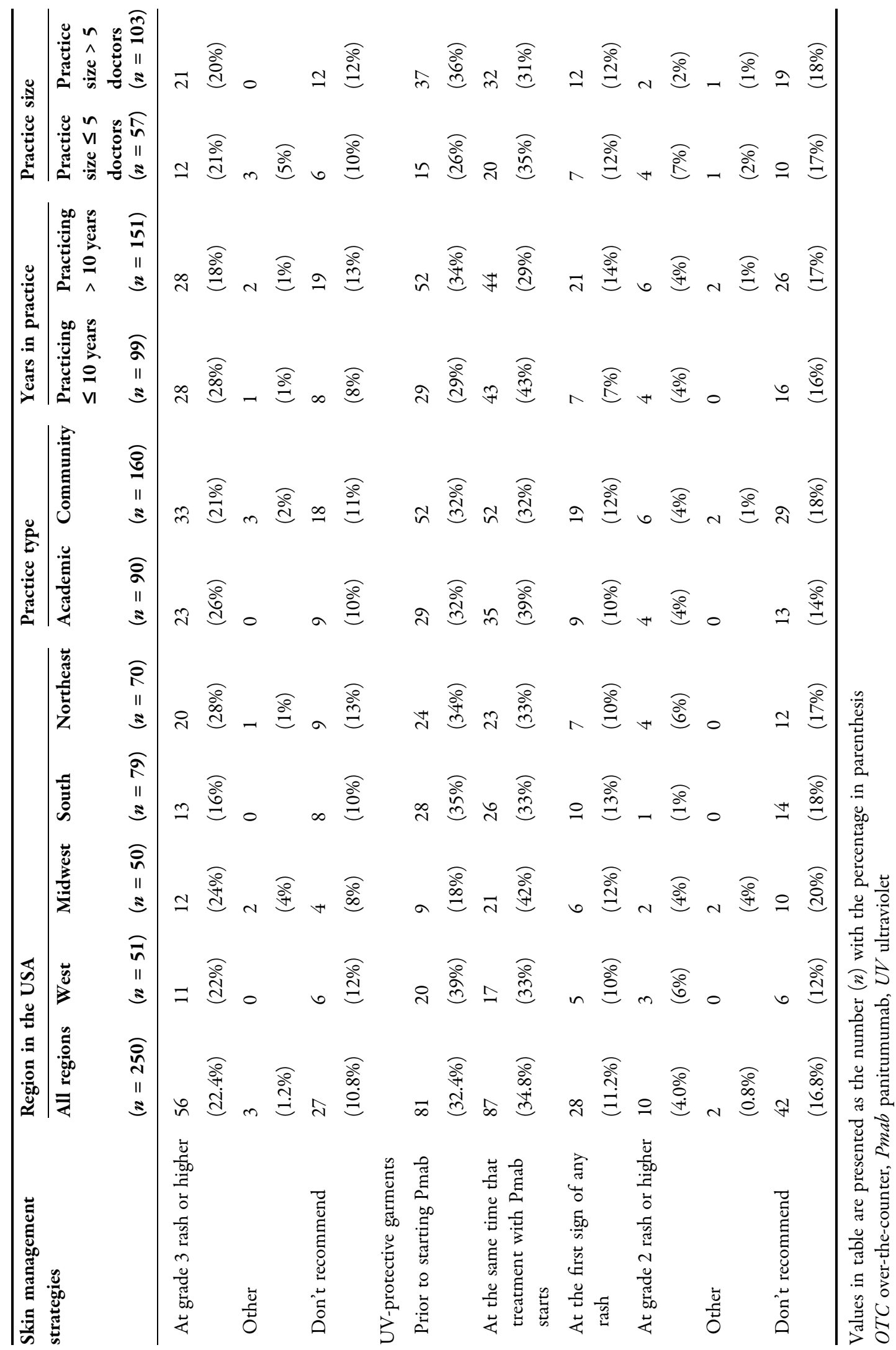




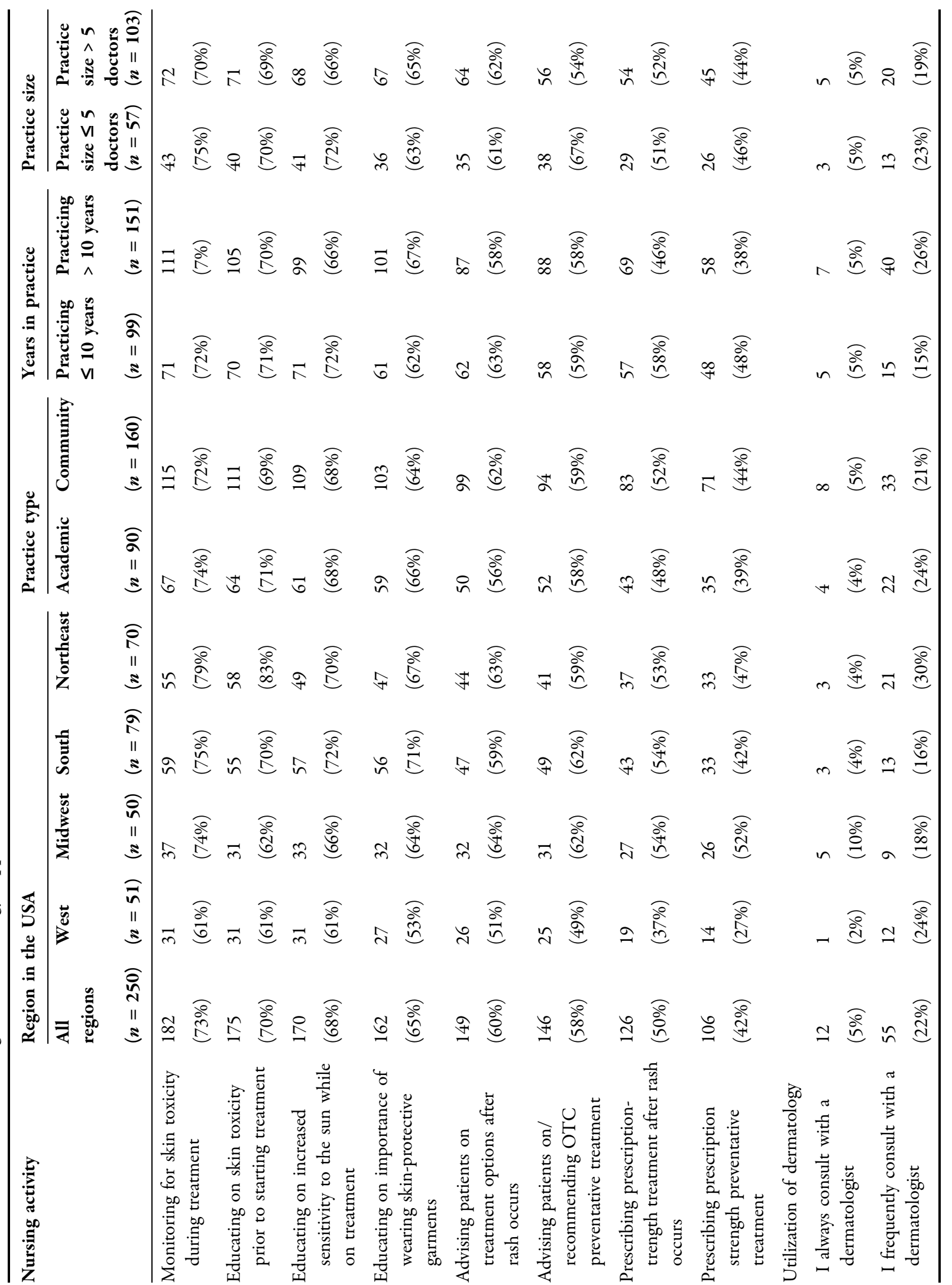




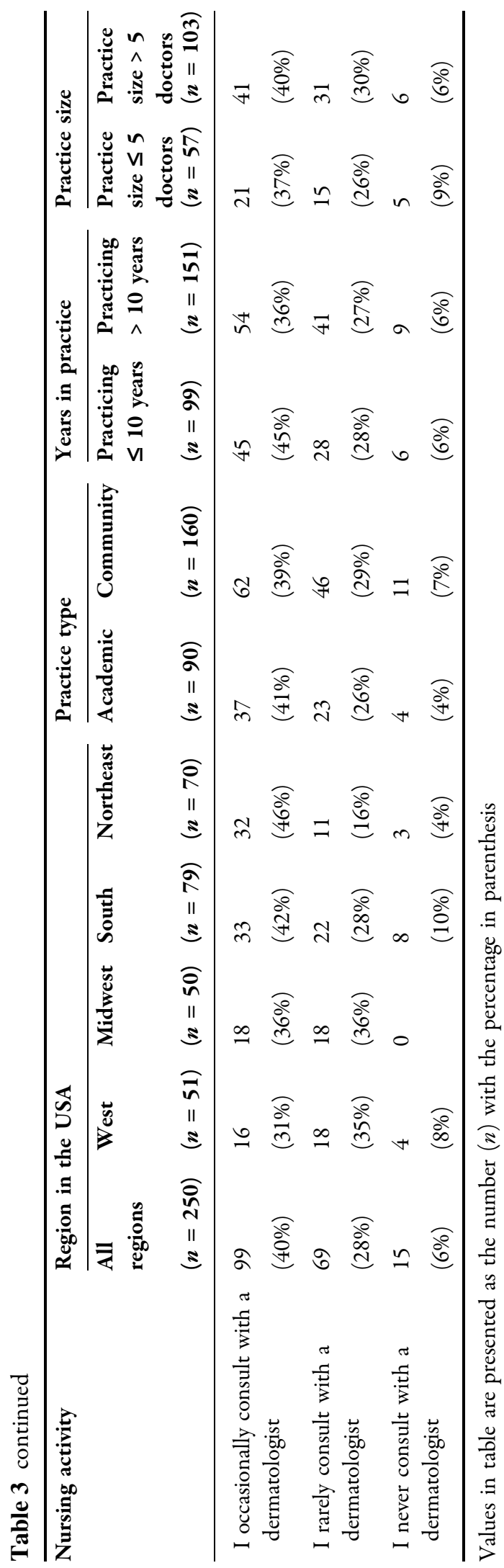

mCRC patients can be greatly improved. In particular, use of moisturizers, sunscreen, and UV-protective garments should be universally recommended to all patients. Our data suggest that approximately $82 \%$ of patients were receiving recommendations for moisturizer, $88 \%$ for sunscreen, and $67 \%$ for UV-protective garments prior to or at the time of the initiation of panitumumab therapy. These aspects of baseline skin care are an effective intervention to improve the integrity of the skin and are an integral part of the strategy to manage cutaneous reactions prior to, during, and after oncology therapy [15].

While the epidemiology of acute dermatologic toxicities are well described in the literature, a large knowledge gap currently exists on the management strategies of these ever-present skin toxicities. The multinational association for supportive care in cancer (MASCC) Skin Toxicity Study Group has developed clinical guidelines for the prevention and treatment of dermatologic toxicities associated with antiEGFR therapies [16]. However, because these guidelines have yet to be incorporated as the standard of care, wide discrepancies exist among providers for how to best prevent and manage skin toxicity and the associated antiEGFR treatment regimens following presentation of rash. The MASCC guidelines include preventive recommendations, such as topical $1 \%$ hydrocortisone cream with moisturizer and sunscreen and systemic treatment with $100 \mathrm{mg}$ of minocycline or doxycycline daily. These preventive recommendations are based in part on regimens found to be effective in the STEPP and J-STEPP studies [11, 12]. The STEPP and J-STEPP studies each demonstrated reduced severity in panitumumab-associated dermatologic toxicities through the implementation of pre-emptive versus reactive skin management. The STEPP study also reported that the qualityof-life measure was less impaired in patients in the pre-emptive group than in patients in the reactive group [11]. In these studies, pre-emptive treatment began 1 day before the first panitumumab dose and continued through weeks $1-6$ of treatment. The regimen consisted of skin moisturizer, sunscreen, $1 \%$ hydrocortisone cream, and and doxycycline $100 \mathrm{mg}$ twice 
per day. The MASCC guidelines are also based on data showing the effectiveness of oral antibiotics to reduce the incidence and severity of rash. These data were recently summarized in a 2016 meta-analysis that reported prophylactic treatment with oral tetracyclines (doxycycline or minocycline) reduced by approximately 50\% the odds of developing a skin rash of any grade (odds ratio [OR] $0.54,95 \%$ confidence interval [CI] $0.40-0.73$ ) and by approximately $70 \%$ the odds of grade 2 or 3 rash (OR $0.36,95 \%$ CI 0.22-0.60) [17].

Despite the positive results from the STEPP and JSTEPP studies, few oncologists in our study followed these strategies, and a high proportion of physicians were not familiar with them. While our data suggest that skin moisturizer and sunscreen are the most commonly recommended treatments, the proportion of patients receiving either intervention can be greatly improved. Recommendations from a multinational expert panel reviewing the evidence for non-pharmaceutical skin care products to prevent and manage skin toxicity resulting from oncology therapies were recently published [15]. The authors stated that "...all anticancer therapy-related cutaneous adverse events are linked to skin barrier dysfunction." Moisturizers were identified as a key component to improve barrier function and skin hydration, thereby reducing pruritus and preventing secondary infection due to scratching. The authors also noted how sun exposure can exacerbate rash resulting from anti-EGFR therapies and, therefore, they recommend the daily application of a broad-spectrum sunscreen. The study further reports on cosmetics and non-pharmaceutical skin care products that may further irritate and thus worsen skin toxicities. To this end, collaborations between oncologists and dermatologists are thought to maximize the management of adverse cutaneous reactions while minimizing changes to therapy $[15,18,19]$. The data from our study reveal that dermatologists were infrequently part of the management team and that their involvement could be increasingly utilized. To the contrary, nurses were heavily utilized as a resource to monitor for and manage skin toxicity in our population. Despite this high frequency of use of nurses, there may still be room for improvement. Nurses may be ideally suited to improve the rates of patient education related to photosensitivity that occurs with anti-EGFR therapies, the importance of sunscreen and of UV-protective garments, as well as the importance of daily moisturizer. Nurses could also provide education on products that should be avoided during the treatment period and during periods of rash.

A few other surveys of treatment providers have been published that describe management practices, among which is one US study [7], one French study [19], and one German study [18]. The results in these studies are similar to our results with regards to the underutilization of dermatology. Few respondents in our study said they always consult with a dermatologist (5\%), whereas it was more common for respondents to say they occasionally (40\%), rarely (28\%), or never $(6 \%)$ consulted a dermatologist. The US study reported only $8 \%$ of respondents obtained a dermatology consult; only $9 \%$ of the German medical oncologists would have referred the case patient to a dermatologist; and the French study reported $97 \%$ of respondents did not consider a routine dermatology consultation at the time of anti-EGFR initiation, while $76 \%$ declared they had never sent their patients to a dermatologist prior to the appearance of skin lesions. While other results were not directly comparable, the results from these three studies do provide useful insight into how providers are managing skin toxicity. Boone et al. conducted an in-person survey among 110 practitioners of US oncology practices using a questionnaire with 51 open-ended questions pertaining to incidence of rash, treatment practices, patient perceptions, and outcome in treating the rash [7]. This US study reported that only $47 \%$ of the respondents actively treated grade 1 rash, 71\% treated grade $2,87 \%$ treated grade 3 , and $80 \%$ treated grade 4 . The rash was noted to be painful by many patients, resulting in $32 \%$ of providers reporting that pain medications were prescribed for patients because of the rash. Discontinuation of EGFR therapy due to rash was common $(32 \%)$, and dose reductions of $10-50 \%$ were reported by $60 \%$ of the providers. The French study was conducted by Peuvrel et al. and consisted of a survey among 67 French 
practitioners related to prophylactic and curative management of EGFR skin toxicities based on a questionnaire with 31 questions covering 11 clinical situations [19]. In addition to the underutilization of dermatologists, this study also reported that facial moisturizer was proposed by $69 \%$ of respondents and doxycycline daily dosage of $100 \mathrm{mg}$ by $66 \%$ of respondents. Only eight practitioners (12\%) advised patients to avoid exposure to the sun [19]. Lastly, the German study implemented a survey among 149 German oncologists (106 medical and 43 dermatological); this study utilized a 7-item questionnaire along with pictures and history of a patient with acneiform rash, and the respondents were asked to provide information regarding grading and treatment strategies [18]. In this study, $22 \%$ of respondents used preemptive treatment. With regard to the case scenario, 91\% chose topical treatment with hydrocortisone or antibiotic cream and 64\% chose systemic treatment with an antibiotic or isotretinoin.

This study has several strengths. First, we included a randomly selected sample of practicing US oncologists derived from a national database that has access to over 2 million physicians. Since the participants were well represented across the USA and reported many years in practice with robust patient loads, we therefore feel our results are likely generalizable to US oncologists who treat mCRC patients with panitumumab. Second, the survey captured real-world data on current practices and opinions of oncologists for managing anti-EGFR skin toxicity. Third, in an attempt to reduce measurement error, the survey went through a rigorous development and validation process which included two rounds of pilot testing prior to implementation.

The study also may have some limitations. The survey sought to capture "usual" practices of oncologists; thus, the extent to which patient-level characteristics influenced treating decisions were not available in this study. If oncologists have widely variable practices that differ by patient demographics, then this study would not capture "usual" practices. Because the survey included physicians treating mCRC patients within the last 3 months, there is potential for recall bias among those providers on the outer limits of these inclusion criteria. However, given the mean number of patients treated with Vectibix in the last 3 months was 22 patients, recall bias is likely to be minimal.

\section{CONCLUSIONS}

These data highlight important gaps in the management strategies related to anti-EGFR skin toxicities. Interventions are currently available to decrease the intensity and severity of dermatologic skin toxicities, resulting in improvements to a patient's quality of life and minimizing treatment interruptions. Specifically, education regarding the pre-emptive use of sunscreen, moisturizers, and UV-protective clothes should be provided to all patients receiving anti-EGFR therapies. However, universal implementation of these strategies will be challenging until they become the standard of care. To expedite that process, multifaceted information campaigns are needed that target the oncologist, nurses, and patients and their support groups. In addition, increasing continuing medical education opportunities on this topic could further improve the knowledge base. Drug representatives could also serve to inform oncologists of best practices for managing rash. The pre-emptive use of OTC topical steroids and oral antibiotics could also be dramatically improved, ideally with the patient receiving all available interventions in agreement with the MASCC recommendations. Among patients with rash, improvements are needed in integrating care with dermatologists. Future research should aim to understand barriers to provision of these management strategies among oncologists and barriers to uptake of these strategies among patients.

\section{ACKNOWLEDGEMENTS}

The authors would like to thank and acknowledge the following people for their contributions to this article: (1) the study participants for their time and contributions to this topic; (2) Human Identity for graphics support. 
Support for this assistance was funded by Amgen, Inc.

Funding. This research project was funded by Amgen, Inc. Amgen was involved in the study design, data collection and analysis, decision to publish, and preparation of the manuscript. All authors had full access to all of the data in this study and take complete responsibility for the integrity of the data and accuracy of the data analysis. The article processing charges were funded by Amgen, Inc.

Authorship. All named authors meet the International Committee of Medical Journal Editors (ICMJE) criteria for authorship for this article, take responsibility for the integrity of the work as a whole, and have given their approval for this version to be published.

Disclosures. Kimberly A. Lowe is an employee of Amgen, Inc. and owns stock in Amgen, Inc. GK is an employee of Amgen, Inc. and owns stock in Amgen, Inc. Tamer Garawin is an employee of Amgen, Inc. and owns stock in Amgen, Inc. Laura Sangare is employed by SimulStat, work contracted through Amgen, Inc. Rachel Bergstresser is employed by Adelphi Research, work contracted through Amgen, Inc. Michelle McNamara is employed by Adelphi Research, work contracted through Amgen, Inc.

Compliance with Ethics Guidelines. All procedures performed in studies involving human participants were in accordance with the Quorum Review institutional review board in the US and with the 1964 Helsinki declaration and its later amendments or comparable ethical standards. Informed consent was obtained from all individual participants included in the study.

Data Availability. The datasets generated during and/or analysed during the current study are available from the corresponding author on reasonable request.

Open Access. This article is distributed under the terms of the Creative Commons Attribution-NonCommercial 4.0 International
License (http://creativecommons.org/licenses/ by-nc/4.0/), which permits any noncommercial use, distribution, and reproduction in any medium, provided you give appropriate credit to the original author(s) and the source, provide a link to the Creative Commons license, and indicate if changes were made.

\section{REFERENCES}

1. Siegel RL, Miller KD, Fedewa SA, et al. Colorectal cancer statistics, 2017. CA Cancer J Clin. 2017;67(3):177-93.

2. Kindler HL, Shulman KL. Metastatic colorectal cancer. Curr Treat Options Oncol. 2001;2(6): 459-71.

3. Kurkjian C, Kummar S. Advances in the treatment of metastatic colorectal cancer. Am J Ther. 2009; 16(5):412-20.

4. Tol J, Punt CJ. Monoclonal antibodies in the treatment of metastatic colorectal cancer: a review. Clin Ther. 2010;32(3):437-53.

5. Torre LA, Bray F, Siegel RL, Ferlay J, Lortet-Tieulent J, Jemal A. Global cancer statistics, 2012. CA Cancer J Clin. 2015;65(2):87-108.

6. Moorcraft SY, Smyth EC, Cunningham D. The role of personalized medicine in metastatic colorectal cancer: an evolving landscape. Therap Adv Gastroenterol. 2013;6(5):381-95.

7. Boone SL, Rademaker A, Liu D, Pfeiffer C, Mauro DJ, Lacouture ME. Impact and management of skin toxicity associated with anti-epidermal growth factor receptor therapy: survey results. Oncology. 2007;72(3-4):152-9.

8. Fakih M, Vincent M. Adverse events associated with anti-EGFR therapies for the treatment of metastatic colorectal cancer. Curr Oncol. 2010;17[Suppl 1]:S18-30.

9. Wagner LI, Lacouture ME. Dermatologic toxicities associated with EGFR inhibitors: the clinical psychologist's perspective. Impact on health-related quality of life and implications for clinical management of psychological sequelae. Oncology (Williston Park). 2007;21[11 Suppl 5]:34-6.

10. Eilers RE Jr, Gandhi M, Patel JD, et al. Dermatologic infections in cancer patients treated with epidermal growth factor receptor inhibitor therapy. J Natl Cancer Inst. 2010;102(1):47-53. 
11. Lacouture ME, Mitchell EP, Piperdi B, et al. Skin toxicity evaluation protocol with panitumumab (STEPP), a phase II, open-label, randomized trial evaluating the impact of a pre-Emptive Skin treatment regimen on skin toxicities and quality of life in patients with metastatic colorectal cancer. J Clin Oncol. 2010;28(8):1351-7.

12. Kobayashi Y, Komatsu Y, Yuki S, et al. Randomized controlled trial on the skin toxicity of panitumumab in Japanese patients with metastatic colorectal cancer: HGCSG1001 study; J-STEPP. Future Oncol. 2015;11(4):617-27.

13. De Tursi M, Zilli M, Carella C, et al. Skin toxicity evaluation in patients treated with cetuximab for metastatic colorectal cancer: a new tool for more accurate comprehension of quality of life impacts. Onco Targets Ther. 2017;10:3007-15.

14. Joshi SS, Ortiz S, Witherspoon JN, et al. Effects of epidermal growth factor receptor inhibitor-induced dermatologic toxicities on quality of life. Cancer. 2010;116(16):3916-23.

15. Bensadoun RJ, Humbert P, Krutman J, et al. Daily baseline skin care in the prevention, treatment, and supportive care of skin toxicity in oncology patients: recommendations from a multinational expert panel. Cancer Manag Res. 2013;5:401-8.

16. Lacouture ME, Anadkat MJ, Bensadoun RJ, et al. Clinical practice guidelines for the prevention and treatment of EGFR inhibitor-associated dermatologic toxicities. Support Care Cancer. 2011;19(8):1079-95.

17. Petrelli F, Borgonovo K, Cabiddu M, et al. Antibiotic prophylaxis for skin toxicity induced by antiepidermal growth factor receptor agents: a systematic review and meta-analysis. $\mathrm{Br} J$ Dermatol. 2016;175(6):1166-74.

18. Hassel JC, Kripp M, Al-Batran S, Hofheinz RD. Treatment of epidermal growth factor receptor antagonist-induced skin rash: results of a survey among German oncologists. Onkologie. 2010;33(3):94-8.

19. Peuvrel L, Bachmeyer C, Reguiai Z, et al. Survey on the management of skin toxicity associated with EGFR inhibitors amongst French physicians. J Eur Acad Dermatol Venereol. 2013;27(4):419-29. 